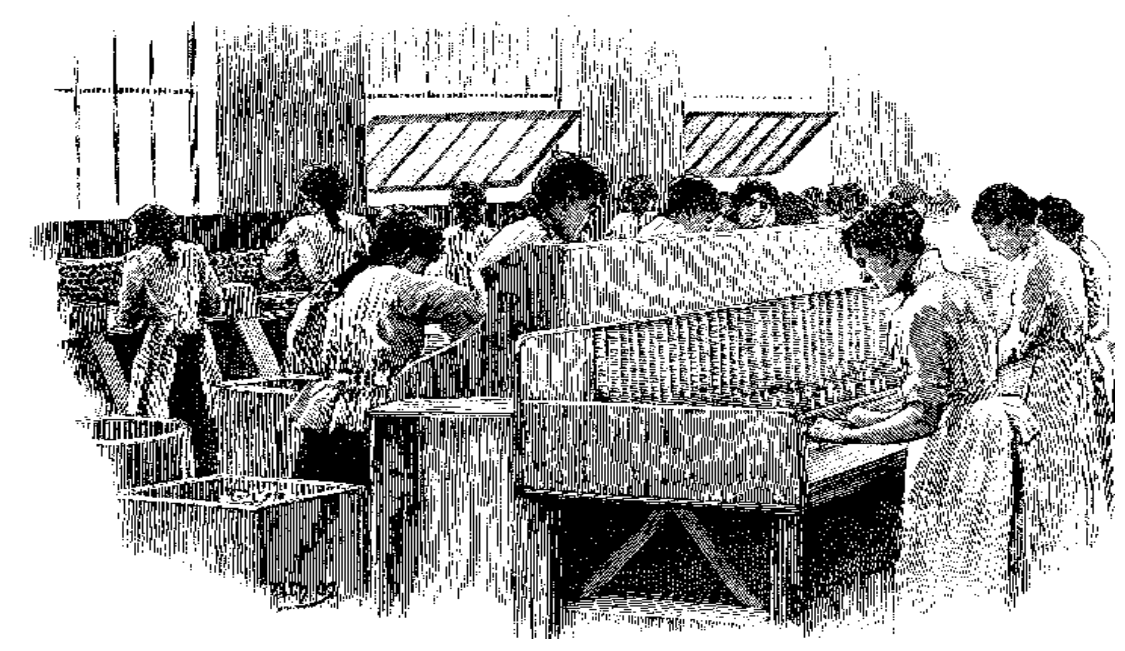

\title{
DEFINICIÓN Y MISIÓN DE LA EDUCACIÓN ESPECIAL ${ }^{1}$
}

\author{
Christiane Lepot-Froment \\ y Michel Mercier
}

\author{
Versión del francés realizada por: Julia Inés Landazábal Q. \\ Profesora Asociada Universidad Pedagógica Nacional \\ María Cristina Melo R. \\ Profesora Titular Universidad Pedagógica Nacional
}

Según una definición muy amplia, se entiende por Educación Especial aquella dirigida y diseñada para los sujetos en situación de discapacidad. Vale la pena preguntarse ¿qué se entiende por discapacidad?

\footnotetext{
${ }^{1}$ Lepot-Froment, Christiane; Mercier, Michel. Introducción al libro: Education Spécialisée. Recherches et pistes d'action. Collection Perspectives en Éducation. Ed. De Boeck et Larcier. Buxelles, 1996: 7-14.
}

Atendiendo a la clasificación internacional sugerida por la Organización Mundial de la Salud $(\mathrm{OMS})^{2}$, dicho término encierra tres dimensiones que se refieren a: las deficiencias, las incapacidades y las desventajas. En algunos casos, las relaciones entre dichas dimensiones son aparentemente

\footnotetext{
${ }^{2}$ Clasificación internacional de las discapacidades: Deficiencias, incapacidades y desventajas (1988). París: Publicación conjuntaOMS/INSERM/CTNERHI.
}

claras, como cuando se habla de la discapacidad relacionada con una deficiencia profunda y precoz: la deficiencia reside en la alteración grave de un órgano (oído interno), la función que dicho órgano ejercería (audición) y puede describirse en términos objetivos (fisiológicos y audiométricos). Esta deficiencia ocasiona incapacidades como la imposibilidad de adquirir el lenguaje oral si no se recurre a procedimientos como la utilización de 
prótesis, o a métodos como la educación audio-oral. Las desventajas debidas tanto a la deficiencia misma como a las incapacidades que de ella se derivan, se refieren en el presente caso a la restricción para el acceso a los recursos culturales del medio, la limitación en las posibilidades de formación y de ejercicio profesional, así como la estigmatización de que puede ser objeto la persona sorda.

Pero estas relaciones son menos claras en otros casos. Por ejemplo en el caso de la discapacidad relacionada con la deficiencia intelectual, la noción de deficiencia plantea el siguiente problema: la naturaleza de la alteración orgánica no es necesariamente conocida, y la referida a la función cognitiva no es 'objetivable' en términos psicométricos. Ahorabien, las apreciaciones psicométricas se llevan a cabo utilizando instrumentos (tests), los cuales no aprehenden sino las funciones intelectuales valoradas socialmente. En otros casos (por ejemplo la dificultad para aprender a leer), la incapacidad implica desventajas en función de las normas impuestas por la sociedad, de la cual hace parte el sujeto juzgado 'incapaz'. Por otra parte, algunas desventajas no pueden ser atribuídas ni a una incapacidad ni a una deficiencia; piénsese en la estigmatización vinculada a características juzgadas como antiestéticas, o que tienen la marca de un grupo étnico menospreciado. Las relaciones consideradas como causales entre deficiencia, incapacidad y desventaja, no se desarrollan en un solo sentido (la deficiencia conlleva la incapacidad, la incapacidad conlleva la desventaja) sino también en el sentido inverso. En efecto, una situación extrema de desventaja (por ej. la pobreza absoluta) donde estaría inmersa una familia, puede acarrear ciertas incapacidades en los miembros que la conforman (para preveer a largo plazo, dominar la expresión simbólica, manejar el pensamiento hipotético); dichas incapacidades, a su vez, inducen al observador armado de 'tests' a concluir en la 'deficiencia' de las funciones cognitivas.

¿Cómo ubicar la acción de la Educación Especial en relación con las dimensiones de la discapacidad? Su misión más obvia consiste en actuar sobre las 'incapacidades'. Pero reducir dichas incapacidades, o remediarlas, exige ineludiblemente apoyarse en las capacidades de la persona. Esto implica transformar la mirada sobre dicha persona: no se trata solamente de determinar sus limitaciones sino ir más allá poniendo en evidencia aque- llo de lo cual es efectivamente capaz.

Una misión menos evidente, pero también esencial, es la intervención en el eje de las desventajas: se trata de reducirlas actuando sobre el medio y sobre la interacción entre éste y la persona. En este sentido, las acciones son múltiples: adecuación de los lugares públicos, adaptación de los puestos de trabajo, aplicación de nuevas tecnologías; en fin, intervenciones destinadas a transformar las actitudes y los comportamientos sociales con respecto a las personas en situación de discapacidad.

Sin embargo, reducir las desventajas ligadas a la situación de discapacidad, equivale a tomar en cuenta las exigencias y valores de la sociedad de la cual forma parte la persona con discapacidad. ¿Significa esto que la Educación Especial debe enfocarse principalmente a la adaptación social

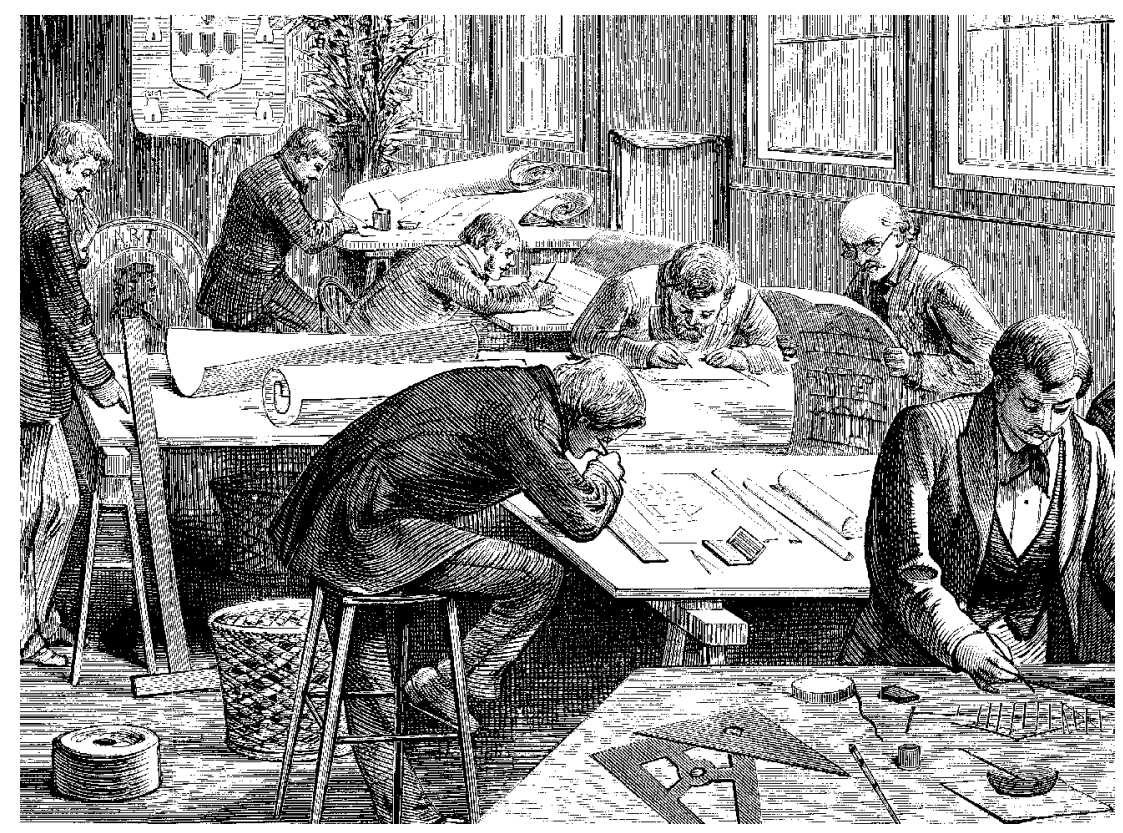




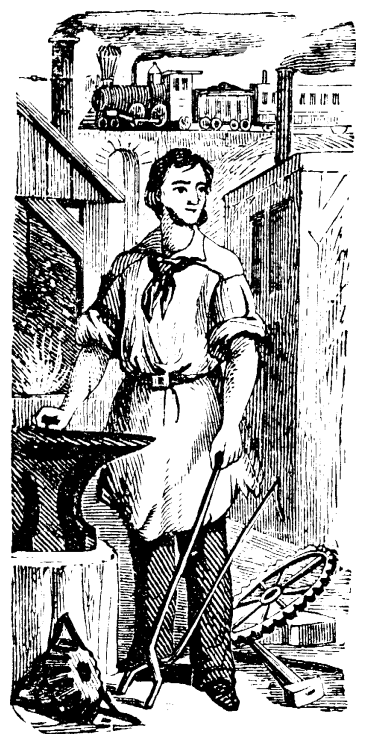

del individuo? Como se dijo antes, la Educación Especial se propone reducir las incapacidades y desarrollar las capacidades de la persona. Sin embargo ¿Cómo decidir entre lo que debe 'reducirse' y aquello que debe 'desarrollarse'? ¿No se trata nuevamente de actuar en función de criterios socialmente determinados? ¿En qué medida se toma en cuenta la realización personal y el bienestar físico y psicológico del individuo?

Se sabe que la misión de la educación general es ayudar a cada individuo a construir las herramientas (afectivas, cognitivas y volitivas) que le permitirán integrarse en la comunidad, trabajar en ella y lograr su realización personal. En la práctica, se tiene frecuentemente la impresión de que los esfuerzos intencionales de entidades educativas como la escuela, se orientan sobre todo a hacer al individuo capaz de responder a las exigencias sociales. En cambio, la misión de ayudar al individuo a lograr competencias que le son propias, no siempre puede explicitarse operacionalmente; los educadores deben determinar si esta misión puede llevarse a cabo y de qué manera lograrlo.

¿Es en el campo de la Educación Especial donde se estima que los niños, adolescentes y adultos, necesitan algo 'diferente' y 'mejor' que el individuo corriente? Una vez más, en el terreno de la práctica, se tiene la impresión de que la selección de las capacidades que deben desarrollarse, está determinada por criterios sociales.

Sin embargo, una de las justificaciones esenciales para legitimar la puesta en práctica de la Educación Especial, es su vocación de individualización, su voluntad de valorar las diferencias. De allí la pregunta: ¿Cómo ayudar a cada individuo a conquistar su estatus de sujeto? Los diferentes actores de la Educación Especial (entre los que están educadores e investigadores), se ven tarde o temprano forzados a plantearse esta pregunta, de manera existencial y no retórica. De ahí que los cuestionamientos se dirijan a la educación en su totalidad.

\section{- El público a quien va dirigida la Educación Especial}

La Educación Especial se dirige a los sujetos en situación de discapacidad o de dificultades de vida temporales. Ahora bien, estas situaciones están diversificadas hasta el infinito así como el público potencial de Educación Especial. Se han propuesto diferentes clasificaciones; además de aquellas que tienen un valor general (como la Clasificación internacional ya citada), existen en los países industrializados clasificaciones que podrían calificarse como administrativas y empleadas para sustentar el derecho de las personas a estructuras adaptadas de educación, alojamiento, trabajo y cuidado. Cada una de estas clasificaciones opera dentro de un abanico (más o menos amplio según el caso) de infinidad de situaciones posibles; los criterios utilizados para operar este abanico conllevan evidentemente una determinación social.

Por otro lado, la puesta en práctica de la Educación Especial, en función de ciertas categorías, implica casi automáticamente la creación de nuevas categorías o sub-categorías.

Por ejemplo, como lo señala la historia de la Educación Especial en los Países Bajos, una enseñanza segregada de los niños con deficiencia auditiva profunda frente a aquellos cuya deficiencia es menos grave, permite evidenciar diferencias entre los niños quienes fueron reagrupados previamente sobre la base de una característica supuestamente homogeneizante (pérdida auditiva de 90 decibeles o más), pero esto no hizo desaparecer sus diferentes actitudes y aptitudes frente a los aprendizajes propuestos. De ahí la creación de 'subcategorías'; por ejemplo la de los 'niños sordos con problemas instrumentales', que llevaría a diferenciarlos de otros niños sordos. Este ejemplo ilustra una tendencia observada frecuentemente en la práctica: aquella que lleva a pensar que la respuesta a la ineludible heterogeneidad de 
todo grupo humano (cualquiera que este sea), reside en la constitución de subgrupos particulares, presumiblemente homogéneos, donde cada uno se situaría más o menos lejos del modelo prototipo del 'buen' sujeto de la educación. Se observa, en efecto, que estas subcategorías se han definido en términos de 'carencias', más que en términos de 'potencialidades'. Es necesario reconocer que no existe la tendencia a crear 'subcategorías positivas' como 'niños sordos dotados para las artes gráficas' o 'niños sordos dotados para el razonamiento matemático'.

Enfrentados, más allá de las etiquetas, a la heterogeneidad de los individuos, la misión de la Educación Especial podría ser diferente: se trataría de reflexionar sobre los objetivos de la individualización pedagógica y buscar los modos y las condiciones para lograrla.

Esta exigencia se impone aún más pues la Educación Especial no se dirige solamente a algunas edades claramente circunscritas. Testimonio de ello es el amplio abanico de lugares en donde tiene aplicación: servicios de estimulación precoz para los bebés discapacitados y sus familias; escuelas (preescolar, primaria y secundaria) ordinarias y especiales; enseñanza superior integrada; centros de formación para jóvenes y adultos.

Tener en cuenta la edad de la persona sujeto de Educación Especial, plantea muchas preguntas. Por una parte, la definición de una edad 'normal' para adquirir tal o cual habilidad valorada socialmente (por ej. la lec-

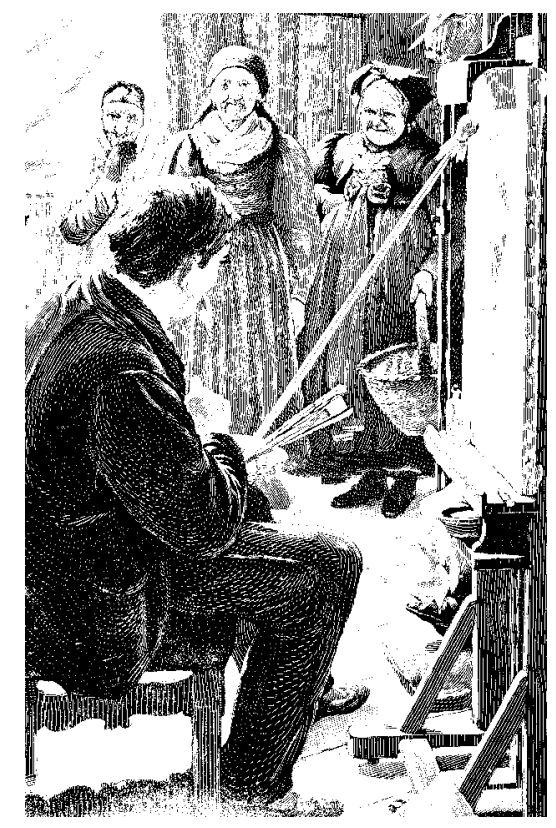

tura) implica la designación del individuo como sujeto de la Educación Especial. Por otra parte, es difícil negar las bondades de la educación precoz, o muy precoz, dirigida a los niños para quienes una deficiencia de origen orgánico y/o condiciones ambientales eminentemente desfavorables, hacen temer que las funciones humanas esenciales, se desarrollen con retardo o carencias. No intervenir, sería j"no ayudar a la persona en peligro"! Y ¿Qué sucede con las personas adultas? En cuanto al individuo común, se admite que la heteroeducación debe dar lugar progresivamente, a la auto-educación. Ahora bien, algunos adultos, y particularmente quienes tienen discapacidad mental, se consideran 'eternos alumnos'. ¿En qué medida es legítima esta actitud? No existe respuesta adecuada a esta pregunta; una respuesta provisional podría ser la de tener en cuenta los deseos y motivaciones de cada individuo.
Algunos adultos prefieren continuar bajo la protección de un mentor, otros están a la búsqueda de un funcionamiento autónomo.

¿Qué pasa con la persona que, en un momento determinado de su historia, recibió Educación Especial por una u otra razón (accidente, enfermedad, traumatismo, avatares sociales)? ¿Cómo será tenida en cuenta esta historia personal, en la propuesta de re-adaptación o de rehabilitación?

La anterior es cuestión importante pues hoy, la Educación Especial no se refiere solamente a las deficiencias tradicionalmente reconocidas. Las crisis sociales hacen surgir nuevas necesidades y emergen nuevas poblaciones: los desempleados, o aquellos que trabajan en exceso. La tendencia es a excluir del mundo laboral a las personas con poca preparación, haciendo recaer en los otros el peso de una competencia que se acrecienta, lo cual plantea nuevos desafíos a la educación.

\section{- Educación Especial y Prevención}

En materia de prevención, se ha hecho clásica la distinción entre prevención primaria (busca prevenir la presencia inicial de la discapacidad), prevención secundaria (tiene que ver con la prevención de discapacidades agregadas a la inicial) y prevención terciaria (orientada a reducir las secuelas o consecuencias negativas de la discapacidad). La educación especial tiene que ver con cada uno de estos campos, aunque los límites entre 
ellos no puedan trazarse con toda claridad. Así, tanto las intervenciones de rehabilitación dirigidas a adultos como la enseñanza especial instituida para los jóvenes, tienen que ver con la prevención terciaria pero, igualmente, con la de nivel secundario.

Finalmente, algunas intervenciones de educación especial, pueden considerarse como acciones de prevención primaria; basta pensar en las campañas de promoción de la salud dirigidas ya sea a un gran público o ya sea a grupos en riesgo, definidos en función de un problema particular. Dichas campañas se proponen modificar actitudes y comportamientos, resultado que sólo puede obtenerse si la educación se apoya en la información.

¿Es necesario definir la prevención como educación especial, o la educación especial como prevención?

\section{- Los Métodos de la Educación Especial}

Los métodos se consideran sobre todo en relación con los contextos escolares (escuelas ordinarias y especiales) pero, según lo señalado en párrafos anteriores, pueden hacerse extensivos a otros contextos.

En la práctica ordinaria de la escuela, no se definen inicialmente unos objetivos individualizados, y se supone que los alumnos deben adaptarse a los métodos que se les proponen. La escuela especial en cuanto tal, tiene que ver claramente con principios de la educación individualizada: los objetivos y métodos se definen para responder a las necesida- des particulares de cada uno de los alumnos. La observación de las prácticas en diversos ámbitos permitiría establecer en qué medida se logra este ideal, cuáles serían las 'disfunciones' posibles y sus posibles soluciones. Sin embargo es necesario llamar la atención sobre dos cuestiones: la primera ha sido nombrada ya y se refiere a la individualización, que no sería sino una falacia si el sujeto que cada uno representa se define sólo en función de un individuo-tipo al cual debe semejarse. Además, la determinación de objetivos individualizados no exime de reflexionar sobre las finalidades que se le asignan a la educación; estas finalidades, que se basan sobre una opción filosófica y una cierta concepción de la existencia humana, ameritan ser formuladas de manera explícita.

¿Qué hay más allá de los métodos de Educación Especial? Un aspecto bastante reconocido es

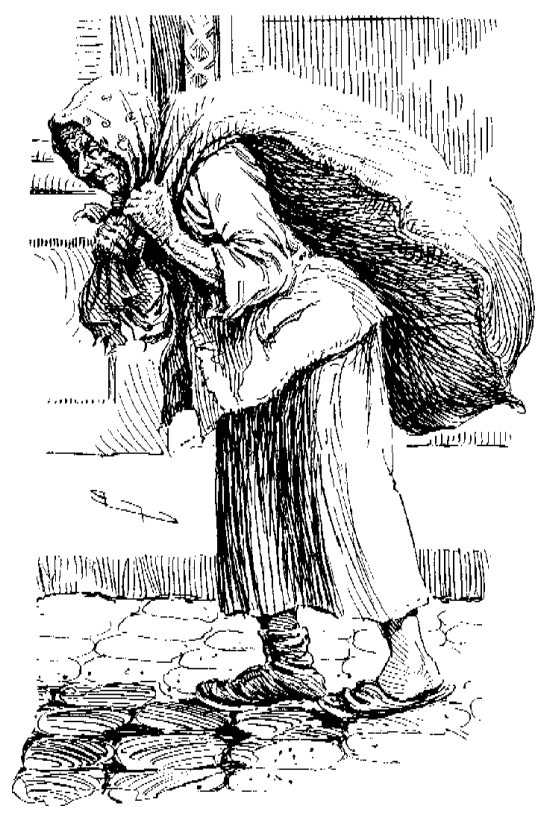

el de la especificidad, no siempre evidente en dichos métodos. Uno de los ejemplos más claros se refiere a los métodos de enseñanza de la lectura que, por diversos que ellos sean, han sido objeto de aplicaciones (más o menos exitosas según el caso), tanto en la enseñanza ordinaria como en la especial. Otros métodos parecen efectivamente específicos y su utilización se reserva para aquellos alumnos que presenten una discapacidad determinada, aunque podrían beneficiar igualmente a alumnos con discapacidad diferente. Esta situación puede también aplicarse al amplio campo de la educación psicomotriz (por no citar sino ésta). En este sentido, podría comprobarse la pertinencia del método verbo-tonal con niños no necesariamente sordos y de la psicomotricidad relacional, aplicada a niños diferentes a aquellos con problemas de la personalidad o del comportamiento. Inversamente, algunos métodos específicos enfocados a ciertas funciones o habilidades aspiran a la categoría de 'método universal'; así, un método de educación auditiva se presenta como la vía para la reconstrucción del equilibrio afectivo, en tanto que otro método de educación del movimiento tiende a definirse como método de educación global.

¿Están los métodos pedagógicos determinados por la naturaleza de la teoría (psicológica, psicofisiológica, psicosocial) a la cual pertenecen sus gestores o sus usuarios? Este punto amerita discusión. La referencia a un modelo teórico explicativo del desarrollo de la persona permite ciertamente comprender mejor aquello que afecta al sujeto en cuestión, y lo 
que está en juego en sus interacciones con el ambiente (social y físico, próximo y remoto). En muchos casos, esta referencia permite igualmente optimizar el proceso en la determinación de los objetivos educativos. Más allá, la selección de los medios que han de utilizarse, pone de manifiesto la creatividad de los educadores: a ellos corresponde deducir las implicaciones de la teoría en la acción pedagógica, valorarlas y ponerlas a prueba. Conviene recordar que, cuando los teóricos formulan sus propuestas educativas, no se fundamentan en otra cosa que no sea la teoría en sí misma; esto es, sobre las intuiciones que les son propias y sobre una determinada concepción de la educación que debe darse al ser humano. No es de extrañar que la referencia a una misma teoría de base engendre opciones educativas diversas; para verificarlo, basta consultar la literatura relativa a las intervenciones pedagógicas de inspiración piagetiana. Puede suceder igualmente, que una práctica pedagógica determinada se alimente en fuentes teóricas diversas, incluso francamente divergentes; por ejemplo,

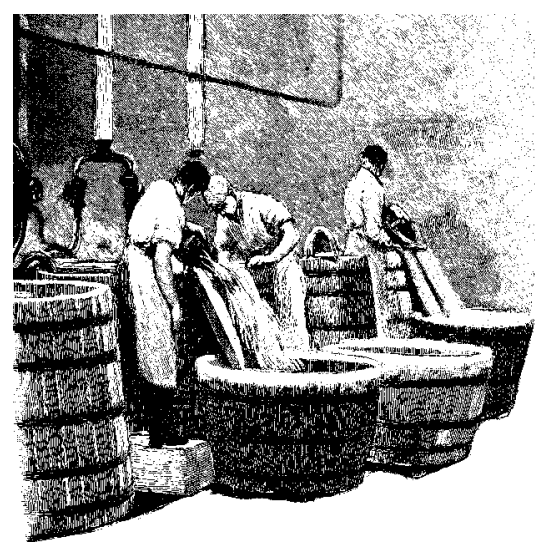

ciertas propuestas educativas se relacionan tanto con Freud como con Piaget. ¿Significa ello una incoherencia del sistema de pensamiento que ha gobernado la concepción de la práctica? Ciertamente no. Las diversas referencias teóricas explicitadas en algunos métodos pedagógicos demuestran, una vez más, que los métodos no pueden reducirse a aplicaciones lineales de una teoría, incluso si el poder explicativo de ésta se revela poderoso. Entre las implicaciones de la teoría y las acciones que ésta podría inspirar, existe un espacio de creatividad cuya importancia no puede subestimarse.

Con respecto a la exigencia de la perspectiva de la individualización, los métodos deben ser utilizados de manera flexible teniendo en cuenta las necesidades específicas de cada alumno. Queda por plantearse la pregunta sobre la legitimidad social de los posibles métodos de intervención. El criterio admitido frecuentemente es el de la 'eficacia'. A partir de ésta y para los seguidores de un método determinado se trata de: (a) Definir los objetivos de manera precisa; (b) Prever el plan cuasiexperimental que permitirá la recopilación sistemática de la información; (c) Comparar los resultados obtenidos con los objetivos previstos. Finalmente, para cumplir con la exigencia de replicabilidad, se comunican los resultados sin omitir la descripción ni del método, ni de las diversas etapas de su implementación. La propuesta clínica comportamental, considerada por algunos como 'enfoque científico' y por otros como 'tecnología', tiene el mérito innegable de resaltar la importancia

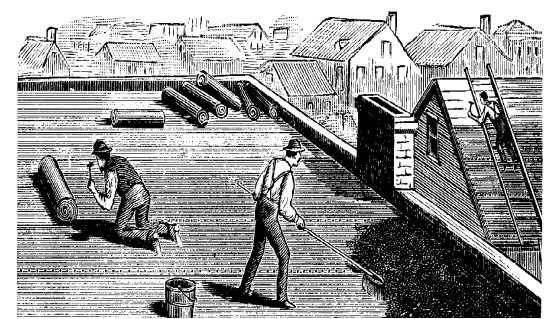

de cada una de sus exigencias y condiciones.

La preocupación por poner a prueba ¿no debería inspirar todos los métodos y los discursos que les subyacen?

Pero, además, ¿la 'puesta a prueba' debería inspirarse solamente por un interés de 'demostración de la eficacia'? En cuanto se refiere a la situación de niños y jóvenes con retardo mental profundo o severo así como con discapacidad motora o sensorial asociada, Jean Jacques Detraux recuerda el imperativo de Saulus ${ }^{3}:$ la percepción débil de los resultados no excluye la obligación de recurrir a los medios que se juzguen necesarios.

La cuestión resaltada por Saulus, a propósito de personas con discapacidad grave es pertinente en otras situaciones: cuando se concluye la ineficacia relativa de una propuesta pedagógica, ¿se está seguro de haber recurrido al conjunto de recursos posibles? Esta pregunta es indispensable puesto que es imposible juzgar a priori la educabilidad de la persona, aún de la más discapacitada, a quien se dirige la acción.

\footnotetext{
${ }^{3}$ Saulus, G. (1987). Prélude Absurde: clin d'oeil a Albert Camus. Besancon. Actes du Colloque A.I.R.
} 